\title{
Who will desire upper airway stimulation as a treatment of obstructive sleep apnea in the Japanese patient population?
}

\author{
Takatoshi Kasai ${ }^{1,2,3,4,5}$
}

Published online: 2 September 2020

(c) Japanese Society of Sleep Research 2020

Obstructive sleep apnea (OSA) is a common chronic condition [1], observed in 10-20\% of the general population [2]. Current population-based studies, including data from Japan, suggested that in addition to men, a substantial proportion of women, especially post-menopausal women, have OSA [2]. Continuous positive airway pressure (CPAP) therapy has been an established treatment option and generally considered as a first-line treatment for moderate to severe OSA. Nowadays, more than 400,000 patients in Japan with OSA are using CPAP. However, several patients with OSA underuse or even discontinue CPAP therapy, and reportedly, 29-83\% were poorly adherent [3]. Although a recent big data analysis in the US found increasing adherence over time (up to $87 \%$ adherent), characteristics, the severity of OSA, and presence or absence of sleepiness are considered as important factors for CPAP adherence [3]. Another option for the treatment of OSA includes use of a mandibular advancement device (MAD). However, the efficacy of MAD on the suppression of OSA varies [4]. Although adherence to MAD use is generally considered better than that to CPAP therapy [5], adherence to MAD use is also highly variable ranging from 32 to $82 \%$ at 1 year [5]. Therefore, effective alternatives

Takatoshi Kasai

kasai-t@mx6.nisiq.net

1 Department of Cardiovascular Medicine, Juntendo University Graduate School of Medicine, 2-1-1 Hongo, Bunkyo-ku, Tokyo 113-8421, Japan

2 Cardiovascular Respiratory Sleep Medicine, Juntendo University Graduate School of Medicine, Tokyo, Japan

3 Department of Cardiovascular Management and Remote Monitoring, Juntendo University Graduate School of Medicine, Tokyo, Japan

4 Department of Digital Health and Telemedicine R\&D, Faculty of Health Science, Juntendo University, Tokyo, Japan

5 Sleep and Sleep Disordered Breathing Center, Juntendo University Hospital, Tokyo, Japan that can successfully suppress OSA and maintain adherence are needed.

Upper airway stimulation (UAS) may be one such alternative for preventing upper airway collapse [6]. The concept of UAS was proposed over 30 years ago [7], but UAS via hypoglossal nerve stimulation recently came into use clinically and will be available in Japan. Hypoglossal nerve stimulation aims to provide electrical stimulation through the programmable generator implanted subcutaneously on the chest wall to activate the muscles to keep the upper airway open during sleep without wearing a nasal/oronasal mask and any other oral appliances [6]. In the US and European countries, UAS using a hypoglossal nerve stimulation device has been approved for several years, and the efficacy of UAS was prominent, especially in less obese patients. [6] As patients do not need to wear masks and oral appliances during sleep, adherence may not be an issue with UAS. Thus, UAS can be an effective alternative, especially in patients with OSA who failed to comply with CPAP and MAD treatment. On the other hand, owing to the requirement of invasive procedures, and based on our clinical experience, we do not know whether patients with OSA in Japan desire UAS.

In this issue, Yamamoto et al. [8] provides insights into the potential of UAS as an alternative to CPAP in Japan. In their study, $20.8 \%$ of their patients with OSA who were using CPAP were willing to be treated with UAS. This is a relatively high proportion than expected; however, it may reflect dissatisfaction with CPAP treatment, independent of its efficacy for suppressing OSA. The reasons why they were willing to be treated with UAS were the inconvenience of carrying the CPAP device followed by discomfort caused by CPAP treatment. The former may be resolved by the replacement with smaller CPAP devices, which have recently come into clinical use. However, the latter reflects the real need for alternatives. In contrast, $56.5 \%$ of them did not want to be treated with UAS. As anticipated, the reasons why they were unwilling to be treated with UAS were the hesitation about having a device implanted into their body, followed 
by satisfaction with their ongoing CPAP treatment. Furthermore, the preference in terms of adherence to CPAP was affected only in female patients, (i.e. CPAP usage at night exceeding $4 \mathrm{~h}$ ) even in the multivariable analysis, suggesting that adherent women may be satisfied with their ongoing therapy and strongly preferred to continue using CPAP than men. In other words, women with poor adherence are likely to be willing to undergo an alternative treatment such as UAS. These findings suggested that there may be a substantial number of patients, especially women with poor adherence to CPAP, who can be potential candidates for UAS in the Japanese OSA patient population. More importantly, these findings remind us of the need for alternative treatments in such patients.

Several limitations leave open questions regarding the interpretation of these findings. First, in their study, although a trained technician blinded to the patient information, explained the details regarding UAS including expected indications in Japan, procedures, adverse events, estimated cost, and actual usage, explanations based on their real clinical experiences may result in different responses. Thus, a relatively higher proportion of patients willing to be treated with UAS should be interpreted with caution. Similar surveillance after encountering several clinical cases of UAS may warrant preference for UAS in the Japanese OSA patient population. Second, if information regarding MAD as another alternative was provided along with the details regarding UAS, some patients may have been willing to be treated with MAD rather than with UAS. In particular, women with poor adherence to CPAP may not prefer UAS but MAD if detailed information regarding MAD was provided with the information regarding UAS. Therefore, results from the surveillance providing information regarding MAD will be more applicable in the real clinical setting. Third, their study lacks information regarding the socioeconomic status; because it was reported that low socioeconomic status is a determinant of CPAP acceptance in patients with OSA [9], this may also affect the preference for UAS. As the exact cost for UAS has yet not been determined in Japan, the effects of socioeconomic status on the preference for UAS should be taken into account for future post-marketing surveillance. Finally, because these findings were from the surveillance in a single academic sleep center, patients enrolled in their study may not represent the typical Japanese OSA patient population. Thus, a multicenter study or national surveillance is required to draw a solid conclusion.

Despite these limitations, the study by Yamamoto et al. provided novel and important information regarding the potential of UAS in the Japanese OSA patient population. We are looking forward to seeing surveillance after the official approval for its clinical use under the Japanese health insurance system.

Funding None.

\section{Compliance with ethical standards}

Conflict of interest T. Kasai is affiliated with departments endowed by Philips Respironics, ResMed, Teijin Home Healthcare and Fukuda Denshi, Paramount Bed, Philips, Asahi Kasei, InterReha, and Toho Holdings.

\section{References}

1. Taranto Montemurro L, Kasai T. The upper airway in sleep-disordered breathing: UA in SDB. Minerva Med. 2014;105:25-40.

2. Matsumoto T, Chin K. Prevalence of sleep disturbances: sleep disordered breathing, short sleep duration, and non-restorative sleep. Respir Investig. 2019;57:227-37.

3. Weaver TE, Grunstein RR. Adherence to continuous positive airway pressure therapy: the challenge to effective treatment. Proc Am Thorac Soc. 2008;5:173-8.

4. Marklund M, Braem MJA, Verbraecken J. Update on oral appliance therapy. Eur Respir Rev. 2019;28:190083.

5. Sunwoo BY, Light M, Malhotra A. Strategies to augment adherence in the management of sleep-disordered breathing. Respirology. 2020;25:363-71.

6. Yamauchi M, Satoh M, Kitahara T, Ota I, Strohl K. Nerve stimulation for the treatment of obstructive sleep apnea. Sleep Biol Rhythms. 2020;18:77-87.

7. Hida W, Okabe S, Miki H, Kikuchi Y, Taguchi O, Takishima $\mathrm{T}$, et al. Effects of submental stimulation for several consecutive nights in patients with obstructive sleep apnoea. Thorax. 1994;49:446-52.

8. Yamamoto U, Soda S, Fujita K, Sawatari H, Ando S. Gender differences in the preference for upper airway stimulation therapy among Japanese patients with obstructive sleep apnea already treated with continuous positive airway pressure. Sleep Biol Rhythms. 2020. https://doi.org/10.1007/s41105-020-00273-x.

9. Simon-Tuval T, Reuveni H, Greenberg-Dotan S, Oksenberg A, Tal A, Tarasiuk A. Low socioeconomic status is a risk factor for CPAP acceptance among adult OSAS patients requiring treatment. Sleep. 2009;32:545-52.

Publisher's Note Springer Nature remains neutral with regard to jurisdictional claims in published maps and institutional affiliations. 